\title{
Neonatal Septicemia in Nepal: Early-Onset versus Late-Onset
}

\author{
Shamshul Ansari, Hari Prasad Nepal, Rajendra Gautam, Sony Shrestha, \\ Puja Neopane, and Moti Lal Chapagain
}

Department of Microbiology, Chitwan Medical College, Bharatpur, Chitwan, Nepal

Correspondence should be addressed to Shamshul Ansari; shamshulansari483@yahoo.com

Received 4 August 2015; Accepted 4 November 2015

Academic Editor: Raymond J. Hutchinson

Copyright (C) 2015 Shamshul Ansari et al. This is an open access article distributed under the Creative Commons Attribution License, which permits unrestricted use, distribution, and reproduction in any medium, provided the original work is properly cited.

\begin{abstract}
Introduction. Neonatal septicemia is defined as infection in the first 28 days of life. Early-onset neonatal septicemia and late-onset neonatal septicemia are defined as illnesses appearing from birth to three days and from four to twenty-eight days postnatally, respectively. Methods. In this cross-sectional study, blood samples from the suspected infants were collected and processed in the bacteriology laboratory. The growth was identified by standard microbiological protocol and the antibiotic sensitivity testing was carried out by modified Kirby-Bauer disk diffusion method. Results. Among total suspected cases, the septicemia was confirmed in $116(12.6 \%)$ neonates. Early-onset septicemia (EOS) was observed in 82 infants and late-onset septicemia (LOS) in 34 infants. Coagulase-negative staphylococcus (CoNS) (46.6\%) was the predominant Gram-positive organism isolated from EOS as well as from LOS cases followed by Staphylococcus aureus (14.6\%). Acinetobacter species (9.5\%) was the predominant Gram-negative organism followed by Klebsiella pneumoniae (7.7\%). Conclusions. The result of our study reveals that the CoNS, Staphylococcus aureus, Acinetobacter spp., and Klebsiella pneumoniae are the most common etiological agents of neonatal septicemia. In particular, since rate of CoNS causing sepsis is alarming, prompting concern to curb the excess burden of CoNS infection is necessary.
\end{abstract}

\section{Introduction}

Septicemia in neonates refers to generalized bacterial infection documented by a positive blood culture in the first 4 weeks of life [1]. Septicemia in neonates can lead to sepsis that is a clinical syndrome characterized by systemic signs of infection and accompanied by bacteremia [2]. Sepsis occurring in the first 72 hours of life is defined as early-onset sepsis (EOS) and that occurring beyond 72 hours as late-onset sepsis (LOS) [3].

Despite advances in health care, neonatal sepsis, and especially that caused by Gram-negative rod bacteria, is a significant cause of morbidity and mortality among neonates [4]. An increase in sepsis caused by Gram-negative organisms has been reported in recent years from Nepal $[5,6]$. Neonatal sepsis caused by Gram-negative microorganisms is responsible for $18 \%-78 \%$ of all neonatal sepsis [7-11]. In the developing world, Escherichia coli (E. coli), Klebsiella species, and Staphylococcus aureus (S. aureus) are the most common pathogens of EOS, whereas S. aureus, Streptococcus pneumoniae, and Streptococcus pyogenes are the most commonly reported organisms in LOS $[12,13]$. Moreover, the causative organisms of EOS and LOS sepsis are similar especially in hospital setting in developing country [14].

Microorganisms implicated in neonatal septicemia have developed increased drug resistance to commonly used antibiotics and thus making treatment extremely difficult [15]. Thus, the knowledge of both the common pathogens causing septicemia in neonates and their antimicrobial susceptibility is essential in order to select appropriate antimicrobial treatment. Moreover, antimicrobial susceptibility patterns of pathogens vary geographically and are temporally dependent on local pathogens and patterns of antibiotic used. Hence, the present study was conducted to document the bacteriological profile of neonatal septicemia and their antibiotic susceptibility profile for planning strategy for the management of neonatal septicemia.

\section{Methods}

A cross-sectional study was carried out from January 2012 to December 2013 at Chitwan Medical College Teaching 
Hospital (a 600-bed hospital) which is located at Bharatpur, Chitwan District of Nepal.

2.1. Study Population. A total of 918 febrile subjects aged up to 28 days with clinical features such as respiratory distress, poor feeding, lethargy, abdominal distension, apnea, irritability, vomiting, convulsions, and cyanosis suggestive of septicemia were enrolled in this study.

2.2. Sample Collection. One milliliter ( $\mathrm{mL})$ of blood samples were collected aseptically by clinicians or trained nurse using sterile syringe and needle by venipuncture and immediately the blood samples were carefully transferred into $9 \mathrm{~mL}$ of Brain Heart Infusion (BHI) broth and labeled with the patient's name, age/sex, identification number, date, and time of collection.

2.3. Bacteriological Processing. The BHI broth inoculated with blood sample was transported to the laboratory and incubated at $37^{\circ} \mathrm{C}$ in aerobic condition. Subcultures were made into sheep blood agar, chocolate agar, and MacConkey agar after overnight of aerobic incubation. Blood agar and MacConkey agar plates were incubated overnight at $37^{\circ} \mathrm{C}$ in aerobic atmosphere while chocolate agar plates were incubated overnight at $37^{\circ} \mathrm{C}$ in $5 \% \mathrm{CO}_{2}$ atmosphere. Thereafter, culture bottles were observed for turbidity for up to 10 days. Final blind subcultures were done before reporting the sample negative. Growth obtained was identified by standard methods [16]. A purity plate was employed to ensure that the inoculum used for the biochemical tests was pure.

2.4. Antibiotic Susceptibility Testing. All the isolates grown were subjected to antibiotic susceptibility testing by modified Kirby-Bauer disk diffusion method in compliance with Clinical and Laboratory Standards Institute (CLSI) guidelines using Mueller-Hinton agar standard media. The inhibition zone standards for antimicrobial susceptibility were considered from tables for interpretative zone diameters of CLSI [17].

Antibiotic disks (HiMedia Laboratories, Pvt. Limited, India) used were oxacillin $(1 \mu \mathrm{g})$, erythromycin $(15 \mu \mathrm{g})$, clindamycin $(2 \mu \mathrm{g})$, vancomycin $(30 \mu \mathrm{g})$, teicoplanin $(30 \mu \mathrm{g})$, penicillin $(10 \mathrm{U})$, cephalexin $(30 \mu \mathrm{g})$, cotrimoxazole $(25 \mu \mathrm{g})$, gentamicin $(10 \mu \mathrm{g})$, amikacin $(30 \mu \mathrm{g})$, ofloxacin $(5 \mu \mathrm{g})$, cefixime $(5 \mu \mathrm{g})$, cefotaxime $(30 \mu \mathrm{g})$, ceftazidime $(30 \mu \mathrm{g})$, piperacillin $(100 \mu \mathrm{g})$, piperacillin-tazobactam $(100 / 10 \mu \mathrm{g})$, carbenicillin (100 $\mu \mathrm{g})$, and ampicillin (10 $\mu \mathrm{g})$.

Staphylococcus aureus ATCC 25923 and Escherichia coli 25922 were used as a control organisms for antibiotic sensitivity testing.

2.5. Ethical Aspects. This study was approved by the Institutional Review Committee of Chitwan Medical College, Bharatpur, Nepal. Informed consent was obtained from the guardians of participating infants before collecting the specimens.
TABLE 1: Sex-wise distribution of total and septicemic cases.

\begin{tabular}{lcccc}
\hline \multirow{2}{*}{ Sex } & \multirow{2}{*}{ Total cases (\%) } & \multicolumn{3}{c}{ Positive cases (\%) } \\
& & Early-onset & Late-onset & Total cases (\%) \\
\hline Male & $564(61.4)$ & $59(72)$ & $22(64.7)$ & $81(69.8)$ \\
Female & $354(38.6)$ & $23(28)$ & $12(35.3)$ & $35(30.2)$ \\
\hline Total & $\mathbf{9 1 8 ( 1 0 0 )}$ & $\mathbf{8 2 ( 1 0 0 )}$ & $\mathbf{3 4 ( 1 0 0 )}$ & $\mathbf{1 1 6 ( 1 0 0 )}$ \\
\hline
\end{tabular}

TABLE 2: Distribution of isolated organisms.

\begin{tabular}{lccc}
\hline & \multicolumn{3}{c}{ Frequency } \\
Organism isolated & $\begin{array}{c}\text { Early-onset } \\
\text { (EOS) }\end{array}$ & $\begin{array}{c}\text { Late-onset } \\
\text { (LOS) }\end{array}$ & Total (\%) \\
\hline Gram-positive organisms & $\mathbf{6 0}$ & $\mathbf{1 4}$ & $\mathbf{7 4}(\mathbf{6 3 . 8})$ \\
S. aureus & 12 & 5 & $17(14.6)$ \\
CoNS & 45 & 9 & $54(46.6)$ \\
Enterococcus spp. & 2 & 0 & $2(1.7)$ \\
Viridans streptococci & 1 & 0 & $1(0.85)$ \\
Gram-negative organisms & $\mathbf{2 2}$ & $\mathbf{2 0}$ & $\mathbf{4 2}(\mathbf{3 6 . 2})$ \\
Acinetobacter spp. & 7 & 4 & $11(9.5)$ \\
Pseudomonas aeruginosa & 5 & 1 & $6(5.2)$ \\
Citrobacter spp. & 1 & 1 & $2(1.7)$ \\
E. coli & 0 & 4 & $4(3.4)$ \\
Enterobacter spp. & 1 & 5 & $6(5.2)$ \\
Klebsiella pneumoniae & 6 & 3 & $9(7.7)$ \\
Burkholderia spp. & 2 & 0 & $2(1.7)$ \\
Proteus mirabilis & 0 & 2 & $2(1.7)$ \\
\hline Total & $\mathbf{8 2}$ & $\mathbf{3 4}$ & $\mathbf{1 1 6 ( 1 0 0 )}$ \\
\hline
\end{tabular}

\section{Results}

3.1. Gender-Wise Distribution of Cases. Of total 918 enrolled neonates, $61.4 \%$ were males and $38.6 \%$ were females with male to female ratio of $1.59: 1$. Among total enrolled cases, septicemia was confirmed in $116(12.6 \%)$ neonates among which EOS was found in $82(70.7 \%)$ neonates while LOS was found in $34(29.3 \%)$ neonates (Table 1).

3.2. Isolates Distribution. Among a total of 116 bacterial isolates recovered, $74(63.8 \%)$ were Gram-positive isolates and 42 (36.2\%) were Gram-negative isolates. Of total positive cases, CoNS were recovered from nearly half of the cases (46.6\%) followed by S. aureus (14.6\%), Acinetobacter spp. (9.5\%), and Klebsiella pneumoniae (7.7\%) whereas viridans streptococci was recovered from a single case. E. coli and Proteus mirabilis were recovered from LOS cases but not from EOS cases while Enterococcus spp., viridans streptococci, and Burkholderia spp. were recovered from EOS case but not from LOS cases (Table 2).

3.3. Antibiotic Resistance Characteristics of Isolates Recovered from EOS Septicemia. Vancomycin and teicoplanin showed $100 \%$ efficacy against Gram-positive isolates. Most of the Gram-positive isolates were resistant to penicillin, 
TABLE 3: Resistance rates of isolates recovered from early-onset cases.

\begin{tabular}{|c|c|c|c|c|c|c|c|c|c|c|c|c|c|c|c|c|}
\hline \multirow{2}{*}{ Isolated organisms $(N)$} & \multicolumn{16}{|c|}{ Resistance rates to different antibiotic tested } \\
\hline & OX & $\mathrm{E}$ & $\mathrm{CD}$ & $\mathrm{P}$ & CFX & COT & G & $\mathrm{AK}$ & OF & CFM & CTX & CAZ & PI & PIT & CAR & AMP \\
\hline S. aureus (12) & 33.3 & 83.3 & 50 & 91.7 & 66.7 & 75 & 58.3 & 25 & 41.6 & - & - & - & - & - & - & - \\
\hline CoNS (45) & 15.5 & 44.4 & 31.1 & 60 & 62.2 & 57.8 & 48.9 & 11 & 29 & - & - & - & - & - & - & - \\
\hline Enterococcus spp. (2) & 0 & 100 & 0 & 100 & 50 & - & 100 & 50 & 50 & - & - & - & - & - & - & - \\
\hline Viridans streptococci (1) & - & 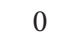 & 0 & 100 & - & 0 & 0 & 0 & 0 & - & - & - & - & - & - & - \\
\hline Acinetobacter spp. (7) & - & - & - & - & - & 14.3 & 28.6 & 14.3 & 28.6 & 42.8 & 28.6 & 57.1 & 57.1 & 42.8 & - & 85.6 \\
\hline Pseudomonas aeruginosa (5) & - & - & - & - & - & - & 20 & 0 & 20 & 80 & 60 & 20 & 40 & 20 & 20 & - \\
\hline Citrobacter spp. (1) & - & - & - & - & - & 0 & 0 & 0 & 100 & 0 & 0 & 0 & - & - & - & 100 \\
\hline Enterobacter spp. (1) & - & - & - & - & - & 100 & 0 & 0 & 0 & 100 & 100 & 100 & 0 & 0 & - & 100 \\
\hline Klebsiella spp. (6) & - & - & - & - & - & 50 & 50 & 16.7 & 16.7 & 66.7 & 50 & 33.3 & 33.3 & 16.7 & - & 100 \\
\hline Burkholderia spp. (2) & - & - & - & - & - & 50 & 50 & 0 & 50 & 100 & 50 & 0 & 0 & 0 & - & 100 \\
\hline
\end{tabular}

OX: oxacillin, E: erythromycin, CD: clindamycin, P: penicillin, CFX: cephalexin, COT: cotrimoxazole, G: gentamicin, AK: amikacin, OF: ofloxacin, CFM: cefixime, CTX: cefotaxim, CAZ: ceftazidime, PI: piperacillin, PIT: piperacillin-tazobactam, CAR: carbenicillin, and AMP: ampicillin. - : not tested.

TABLE 4: Resistant rates of isolates recovered from late-onset septicemia cases.

\begin{tabular}{|c|c|c|c|c|c|c|c|c|c|c|c|c|c|c|c|c|}
\hline \multirow{2}{*}{ Isolated organisms $(N)$} & \multicolumn{16}{|c|}{ Resistance rates to different antibiotic tested } \\
\hline & OX & $\mathrm{E}$ & $\mathrm{CD}$ & $\mathrm{P}$ & CFX & COT & G & $\mathrm{AK}$ & $\mathrm{OF}$ & CFM & CTX & CAZ & PI & PIT & CAR & AMP \\
\hline S. aureus (5) & 40 & 60 & 40 & 80 & 80 & 40 & 40 & 0 & 20 & - & - & - & - & - & - & - \\
\hline CoNS (9) & 44.4 & 66.7 & 22.2 & 89 & 77.8 & 55.5 & 22.2 & 11.1 & 22.2 & - & - & - & - & - & - & - \\
\hline Acinetobacter spp. (4) & - & - & - & - & - & 75 & 0 & 0 & 0 & 75 & 75 & 75 & 25 & 0 & - & 100 \\
\hline Pseudomonas aeruginosa (1) & - & - & - & - & - & - & 100 & 0 & 0 & 100 & 100 & 0 & 100 & 100 & 0 & - \\
\hline Citrobacter spp. (1) & - & - & - & - & - & 0 & 0 & 0 & 0 & 0 & 0 & 0 & 0 & 0 & - & 100 \\
\hline E. coli (4) & - & - & - & - & - & 50 & 50 & 25 & 25 & 25 & 25 & 25 & 0 & 0 & - & 75 \\
\hline Enterobacter spp. (5) & - & - & - & - & - & 100 & 100 & 80 & 100 & 100 & 100 & 80 & 80 & 60 & - & 100 \\
\hline Klebsiella spp. (3) & - & - & - & - & - & 33.3 & 33.3 & 33.3 & 33.3 & 33.3 & 33.3 & 33.3 & - & - & - & 100 \\
\hline Proteus mirabilis (2) & - & - & - & - & - & 0 & 50 & 0 & 0 & 0 & 0 & 0 & 0 & 0 & - & 100 \\
\hline
\end{tabular}

OX: oxacillin, E: erythromycin, CD: clindamycin, P: penicillin, CFX: cephalexin, COT: cotrimoxazole, G: gentamicin, AK: amikacin, OF: ofloxacin, CFM: cefixime, CTX: cefotaxim, CAZ: ceftazidime, PI: piperacillin, PIT: piperacillin-tazobactam, CAR: carbenicillin, and AMP: ampicillin. - : not tested.

erythromycin, and gentamicin whereas amikacin showed a promising efficacy among tested antibiotics. Among Gramnegative isolates, nearly all the isolates were resistant to ampicillin and most of the isolates were resistant to cefixime and cefotaxime while amikacin was found to be most effective among tested antibiotics (Table 3 ).

3.4. Antibiotic Resistance Characteristics of Isolates Recovered from LOS. Of isolates recovered from LOS cases, most of the Gram-positive isolates were resistant to erythromycin, penicillin, and cephalexin while vancomycin and teicoplanin showed $100 \%$ efficacy and amikacin showed better efficacy among all the antibiotics tested. Among Gram-negative isolates, nearly all the isolates were resistant to ampicillin whereas nearly all Citrobacter spp. and Proteus mirabilis were susceptible to most of the antibiotic tested. Nearly all isolates of Enterobacter spp., up to 50\% E. coli isolates, and 33.3\% of the Klebsiella spp. were resistant to most of the antibiotic tested as elaborated in Table 4.

\section{Discussions}

Sepsis remains one of the most important causes of morbidity and mortality in the newborn despite considerable progress in hygiene, introduction of new antimicrobial agents, and advanced measures for early diagnosis and treatment $[18,19]$.

Of the infants born at a tertiary care center located at Bharatpur of central Nepal between January 2012 and December 2013, the septicemia was suspected in 918 infants. Among them $61.4 \%$ infants were males and $38.6 \%$ were female infants. Similar rate of suspected septicemia in male and female infants was also reported by Karambin and Zarkesh from Iran [20] and Al-Shamahy et al. from Yemen [21].

In our setting, the burden of septicemia among total suspected cases was confirmed in 116 infants by positive blood culture growth giving a prevalence rate of $12.6 \%$ which is a lower rate than previously reported by Khanal et al. [5] from eastern Nepal. The lower rate observed in our study may be due to the multiple changes that have occurred with increasing awareness of prevention of sepsis. These changes 
include prevention of preterm labor, earlier and more aggressive enteral feeding and early discontinuation of vascular catheters, shorter duration of invasive ventilation because of surfactant use, better hand hygiene practices, and better protocols for handling vascular lines [14]. However, similar rate of positivity was also detected by Dagnew et al. from Ethiopia [22], Mhada et al. from Tanzania [23], Karambin and Zarkesh from Iran [20], and Mutlu et al. from Turkey [24].

The microorganisms responsible for neonatal sepsis have changed over time, and they vary markedly from region to region. Prematurity, frequent use of catheters, use of total parenteral nutrition, and frequent antibiotic resistance were all reported as causes of change in the etiology of neonatal sepsis [25].

The impacts of specific etiologic agents on blood stream infected patient outcome are tremendous; blood stream infection increases the mortality rate, prolongs patient stay in an intensive care unit and in the hospital, and leads to increased health care costs $[26,27]$. Among the culture proven cases, bacterial septicemia was observed mostly in male neonates $(69.8 \%)$ in the present study whereas it was confirmed to be $30.2 \%$ in female neonates. Similar finding of septicemia in male and female neonates was also detected by Karambin and Zarkesh [20] and Naher and Khamael from Iraq [28].

Since etiologic agents in neonatal EOS and sometimes LOS are often acquired from mother's genital tract, vaginal cultures in all pregnant women as a screening program and appropriate treatment of positive cases before delivery should be emphasized. Among total septicemia cases EOS was found in $82(70.7 \%)$ neonates and LOS was found in $34(29.3 \%)$ neonates. The result indicated that the incidence of EOS septicemia was more common than LOS which is consistent with other reports from Nepal [6], Iran [29, 30], Iraq [28], Bangladesh [31], and Yemen [21].

Although Gram-positive organisms are the most common causes of nosocomial blood stream infections, Gramnegative bacteremia carries higher risks of severe sepsis, septic shock, and death. Sundaram et al. reported a neonatal mortality rate due to Gram-negative sepsis of $34 \%$ to $55 \%$ [14]. Even in the present study, Gram-positive organism constituted the major group of isolates accounting for $63.8 \%$. The higher proportion of Gram-positive organism in this study corroborates with $74 \%$ reported by Khanal et al. from Nepal [5], 69\% reported by Dagnew et al. from Ethiopia [22], and $68 \%$ reported by Mutlu et al. from Turkey [24].

Early-onset neonatal sepsis is caused by microorganisms acquired from the mother before or during birth (vertically transmitted and perinatally acquired); thus, microorganisms from the maternal genital tract may play an important role in early infection [32]. Among Gram-positive group of organisms CoNS was the most common cause of both EOS and LOS accounting for nearly half of the cases (46.6\%) followed by $S$. aureus (14.6\%). Similar rates of CoNS and S. aureus isolates were also reported by Dagnew et al. from Ethiopia [22], Ozkan et al. from Turkey [25], Karambin and Zarkesh [20], and Ghotaslou et al. [33] from Iran. The interpretation of the CoNS to be a cause of septicemia is a major concern for clinicians and clinical microbiology laboratories. The observation of sepsis symptoms and the number of positive blood cultures usually confirms the decision for therapy. However, in this study, the criteria of multiple blood culture were not applied because we could not go for multiple blood sample collection in early age patients. Among Gramnegative organisms, Acinetobacter spp. (9.5\%) were the most common organism isolated from EOS cases while Enterobacter spp. were the predominant organism from LOS cases.

Neonatal sepsis is a life threatening emergency and thus any delay in treatment may cause death. The knowledge of the etiological organisms as well as their antimicrobial sensitivity profile is necessary for commencement of antibiotic therapy empirically while awaiting blood culture results. The initial empiric antibiotic therapy must therefore be a combination of drugs to cover the prevalent bacterial organisms in that locality.

The present study has shown the sensitivity pattern of the common pathogens isolated from EOS as well as LOS to commonly used antibiotics. Aminoglycosides (gentamicin and amikacin) and quinolones (ofloxacin) were observed to be the most effective antimicrobial agents against both Gram-positive and Gram-negative organisms while $\beta$-lactam antibiotics (ampicillin, penicillin, and cephalosporins) were observed as the least effective ones against them in our hospital. Similar pattern of susceptibility was also reported from Nepal [34], India [35], Tureky [25], and Pakistan [36].

Vancomycin and teicoplanin remained the most effective antibiotics against all the Gram-positive isolates from EOS as well as LOS cases; not a single case of resistant isolate was found against vancomycin and teicoplanin. Similarly, vancomycin was also found as the most effective antibiotic in a study by Komolafe and Adegoke from Nigeria [37] and Desai and Malek from India [35]. All of the Gram-positive organisms isolated from both EOS and LOS cases were also found to be susceptible to vancomycin by Ozkan et al. from Turky [25].

In EOS, minority of Gram-positive and Gram-negative isolates were sensitive to commonly tested antibiotics in the current study. Most of the Gram-positive isolates were resistant to erythromycin and gentamicin. Similar proportion of resistance rate in Gram-positive organisms to common antibiotics was also reported by Gheibi et al. from Iran [30]. Among Gram-negative isolates, nearly all the isolates were resistant to amoxicillin, the result being similar to that reported by Mhada et al. from Tanzania [23]. Relatively, amikacin was found to be fairly effective among the tested antibiotics. Effectiveness of amikacin was also highlighted by the literatures from Tanzania [23] and Turkey [24].

The antimicrobial sensitivity pattern differs in different studies as well as at different times in the same hospital. This is because of emergence of resistant strains as a result of indiscriminate use of antibiotics. In LOS, most of the Grampositive isolates were resistant to erythromycin, penicillin, and cephalexin while amikacin was found to be effective regimen among the tested antibiotics. Among Gram-negative isolates, nearly all the isolates were resistant to ampicillin and the Enterobacter spp., and the predominant Gram-negative organism was resistant to most of the antibiotics tested. Nearly all Gram-negative isolates were also reported to be 
resistant to ampicillin by Desai and Malek from India [35]. Most of the Enterobacter spp. isolates tested by Karambin and Zarkesh in Iran also exhibited resistance to several antibiotics tested [20]. High resistance noted in this study may be primarily attributed to excessive and irrational use of these antibiotics at primary health care facilities and private clinics from which neonates are referred to our center.

\section{Conclusions}

This research study identified CoNS, S. aureus, Acinetobacter spp., and Klebsiella pneumoniae as the predominant etiological agents of bloodstream infection among neonates at CMCTH. Effective prophylactic measures, prompt and accurate diagnoses, and subsequent administration of targeted therapy are vital to curb the excessive burden of the disease. An alarmingly high degree of antibiotic resistance observed calls for an urgent evaluation and development of antibiotic policies and protocols for neonatal sepsis. Future epidemiological and clinical studies are also needed to monitor changes in the microorganisms causing neonatal sepsis.

\section{Conflict of Interests}

The authors declare that there is no conflict of interests regarding the publication of this paper.

\section{Acknowledgments}

The authors are deeply indebted to the neonates and their parents participating in this study. They thank all the laboratory staff of the Bacteriology Department of Chitwan Medical College Teaching Hospital (CMCTH) for their kind support during the performance of laboratory tests.

\section{References}

[1] M. Singh, A. K. Deorari, R. C. Khajuria, and V. K. Paul, "Perinatal \& neonatal mortality in a hospital," The Indian Journal of Medical Research, vol. 94, pp. 1-5, 1991.

[2] J. O. Klein, "Bacteriology of neonatal sepsis," Pediatric Infectious Disease Journal, vol. 9, no. 10, pp. 777-778, 1990.

[3] B. J. Stoll, N. I. Hansen, P. J. Sánchez, and et al, "Early onset neonatal sepsis: the burden of group B streptococcal and E. coli disease continues," Pediatrics, vol. 127, no. 5, pp. 817-826, 2011.

[4] B. J. Stoll, "Infections of the neonatal infant," in Nelson Textbook of Pediatrics, R. E. Behrman, R. M. Kleigman, H. B. Jenson, and B. F. Stanton, Eds., pp. 794-811, Saunders, Philadelphia, Pa, USA, 18th edition, 2007.

[5] B. Khanal, M. Shariff, and M. Deb, "Neonatal septicaemia: a hospital based study in Eastern Nepal," Journal of Nepal Medical Association, vol. 43, no. 155, pp. 231-234, 2004.

[6] N. K. Jain, V. M. Jain, and S. Maheshwari, "Clinical profile of neonatal sepsis," Kathmandu University Medical Journal, vol. 1, no. 2, pp. 117-120, 2003.

[7] R. C. Couto, E. A. A. Carvalho, T. M. G. Pedrosa, Ê. R. Pedroso, M. C. Neto, and F. M. Biscione, "A 10-year prospective surveillance of nosocomial infections in neonatal intensive care units," American Journal of Infection Control, vol. 35, no. 3, pp. 183-189, 2007.

[8] B. J. Stoll, N. Hansen, A. A. Fanaroff et al., "Late-onset sepsis in very low birth weight neonates: the experience of the NICHD Neonatal Research Network," Pediatrics, vol. 110, no. 2, pp. 285291, 2002.

[9] S. Kamath, S. Mallaya, and S. Shenoy, "Nosocomial infections in neonatal intensive care units: profile, risk factor assessment and antibiogram," Indian Journal of Pediatrics, vol. 77, no. 1, pp. 37-39, 2010.

[10] Y.-C. Tseng, Y.-C. Chiu, J.-H. Wang et al., "Nosocomial bloodstream infection in a neonatal intensive care unit of a medical center: a three-year review," Journal of Microbiology, Immunology and Infection, vol. 35, no. 3, pp. 168-172, 2002.

[11] N. Macharashvili, E. Kourbatova, M. Butsashvili, T. Tsertsvadze, L.-A. McNutt, and M. K. Leonard, "Etiology of neonatal blood stream infections in Tbilisi, Republic of Georgia," International Journal of Infectious Diseases, vol. 13, no. 4, pp. 499-505, 2009.

[12] A. K. M. Zaidi, D. Thaver, S. A. Ali, and T. A. Khan, "Pathogens associated with sepsis in newborns and young infants in developing countries," Pediatric Infectious Disease Journal, vol. 28, no. 1, pp. S10-S18, 2009.

[13] K. Mulholland, P. Margolis, K. Mason et al., "Bacterial etiology of serious infections in young infants in developing countries: results of a multicenter study," The Pediatric Infectious Disease Journal, vol. 18, no. 10, supplement, pp. S17-S22, 1999.

[14] V. Sundaram, P. Kumar, S. Dutta et al., "Blood culture confirmed bacterial sepsis in neonates in a north Indian tertiary care center: changes over the last decade," Japanese Journal of Infectious Diseases, vol. 62, no. 1, pp. 46-50, 2009.

[15] F. Motara, D. E. Ballot, and O. Perovic, "Epidemiology of neonatal sepsis at Johannesburg Hospital," Southern African Journal of Epidemiology and Infection, vol. 20, pp. 90-93, 2005.

[16] H. D. Isenbergh, Clinical Microbiology Procedures Handbook, ASM Press, Washington, DC, USA, 2nd edition, 2004.

[17] Clinical and Laboratory Standard Institute (CLSI), Performance Standards for Antimicrobial Susceptibility Testing, CLSI: M100S16, Clinical and Laboratory Standard Institute (CLSI), Wayne, Pa, USA, 2006.

[18] S. P. Gotoff, "Neonatal sepsis and meningitis," in Nelson Textbook of Pediatrics, R. E. Behrman, R. M. Kleigman, and A. M. Arvin, Eds., pp. 528-537, W.B. Saunders, Philadelphia, Pa, USA, 15th edition, 1996.

[19] K. H. Haque, "Infection and Immunity in the newborn," in Forfor and Arneil's Textbook of Pediatrics, A. G. M. Campbell and N. Macintosh, Eds., pp. 273-289, Pearson Professional Limited, 5th edition, 1998.

[20] M.-M. Karambin and M. Zarkesh, "Entrobacter, the most common pathogen of neonatal septicemia in Rasht, Iran," Iranian Journal of Pediatrics, vol. 21, no. 1, pp. 83-87, 2011.

[21] H. A. Al-Shamahy, A. A. Sabrah, A. B. Al-Robasi, and S. M. Naser, "Types of bacteria associated with neonatal sepsis in AlThawra University Hospital, Sana'a, Yemen, and their antimicrobial profile," Sultan Qaboos University Medical Journal, vol. 12, no. 1, pp. 48-54, 2012.

[22] M. Dagnew, G. Yismaw, M. Gizachew et al., "Bacterial profile and antimicrobial susceptibility pattern in septicemia suspected patients attending Gondar University Hospital, Northwest Ethiopia," BMC Research Notes, vol. 6, no. 1, article 283, 2013. 
[23] T. V. Mhada, F. Fredrick, M. I. Matee, and A. Massawe, "Neonatal sepsis at Muhimbili National Hospital, Dar es Salaam, Tanzania; Aetiology, antimicrobial sensitivity pattern and clinical outcome," BMC Public Health, vol. 12, article 904, 2012.

[24] M. Mutlu, Y. Aslan, B. Saygin, G. Yilmaz, G. Bayramoğlu, and I. Köksal, "Neonatal sepsis caused by Gram-negative bacteria in a neonatal intensive care unit: a six years analysis," Hong Kong Journal of Paediatrics, vol. 16, no. 4, pp. 253-257, 2011.

[25] H. Ozkan, M. Cetinkaya, N. Koksal, S. Celebi, and M. Hacimustafaoglu, "Culture-proven neonatal sepsis in preterm infants in a neonatal intensive care unit over a 7 year period: coagulase-negative Staphylococcus as the predominant pathogen," Pediatrics International, vol. 56, no. 1, pp. 60-66, 2014.

[26] A. O. Tziamabos and D. L. Kasper, "Principle and practice of infectious diseases," Frank Polizano Journal, vol. 26, pp. 28102816, 2005.

[27] K. M. Madsen, H. C. Schønheyder, B. Kristensen, and H. T. Sørensen, "Secular trends in incidence and mortality of bacteraemia in a Danish county 1981-1994," APMIS, vol. 107, no. 3, pp. 346-352, 1999.

[28] H. S. Naher and A. B. Khamael, "Neonatal sepsis; the bacterial causes and the risk factors," International Research Journal of Medical Sciences, vol. 1, no. 6, pp. 19-22, 2013.

[29] A. H. Movahedian, R. Moniri, and Z. Mosayebi, "Bacterial culture of neonatal sepsis," Iranian Journal of Public Health, vol. 35, no. 4, pp. 84-89, 2006.

[30] S. Gheibi, Z. Fakoor, M. Karamyyar et al., "Coagulase-negative Staphylococcus; the most common cause of neonatal septicemia in Urmia, Iran," Iranian Journal of Pediatrics, vol. 18, no. 3, pp. 237-243, 2008.

[31] C. H. Rasul, M. A. Hassan, and M. Habibullah, "Neonatal sepsis and use of antibiotic in tertiary care hospital," Pakistan Journal of Medical Sciences, vol. 23, no. 1, pp. 78-81, 2007.

[32] B. M. Kerur, B. V. Bhat, B. N. Harish, S. Habeebullah, and C. U. Kumar, "Maternal genital bacteria and surface colonization in early neonatal sepsis," Indian Journal of Pediatrics, vol. 73, no. 1, pp. 29-32, 2006.

[33] R. Ghotaslou, Z. Ghorashi, and M.-R. Nahaei, "Klebsiella pneumoniae in neonatal sepsis: a 3-year study in the pediatric hospital of Tabriz, Iran," Japanese Journal of Infectious Diseases, vol. 60 , no. 2-3, pp. 126-128, 2007.

[34] R. K. Shrestha, S. K. Rai, L. K. Khanal, and P. K. Manda, "Bacteriological study of neonatal sepsis and antibiotic susceptibility pattern of isolates in Kathmandu, Nepal," Nepal Medical College Journal, vol. 15, no. 1, pp. 71-73, 2013.

[35] K. J. Desai and S. S. Malek, "Neonatal septicemia: bacterial isolates and their antibiotics susceptibility patterns," National Journal of Integrated Research in Medicine, vol. 1, no. 3, pp. 12-15, 2010.

[36] Z. Muhammad, A. Ahmed, U. Hayat et al., "Neonatal sepsis: causative bacteria and their resistance to antibiotics," Journal of Ayub Medical College Abbottabad, vol. 22, no. 4, pp. 33-36, 2010.

[37] A. O. Komolafe and A. A. Adegoke, "Incidence of bacterial septicaemia in Ile-Ife metropolis, Nigeria," Malaysian Journal of Microbiology, vol. 4, no. 2, pp. 51-61, 2008. 


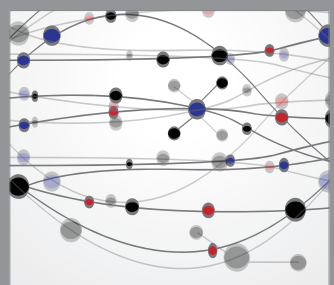

The Scientific World Journal
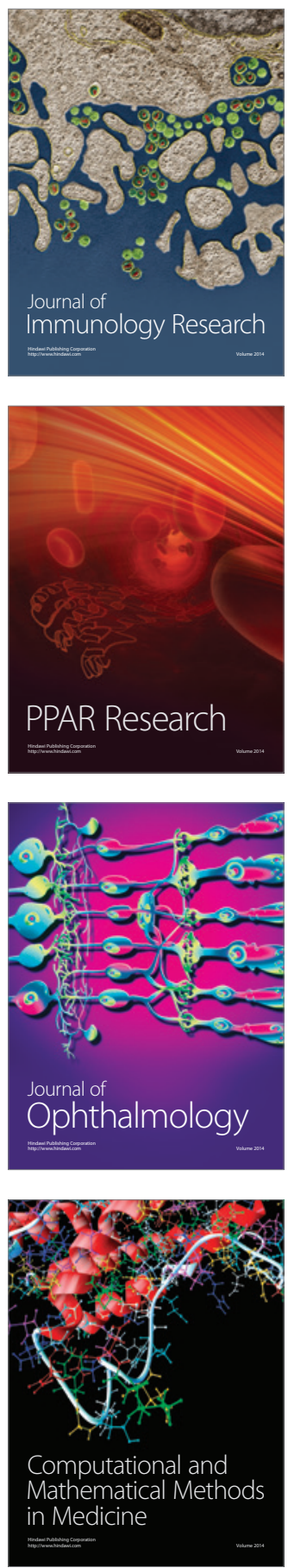

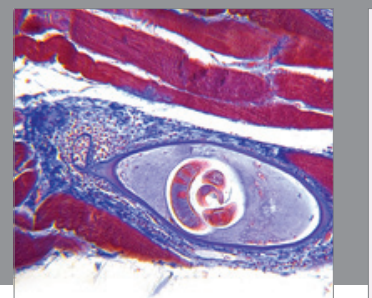

Gastroenterology

Research and Practice
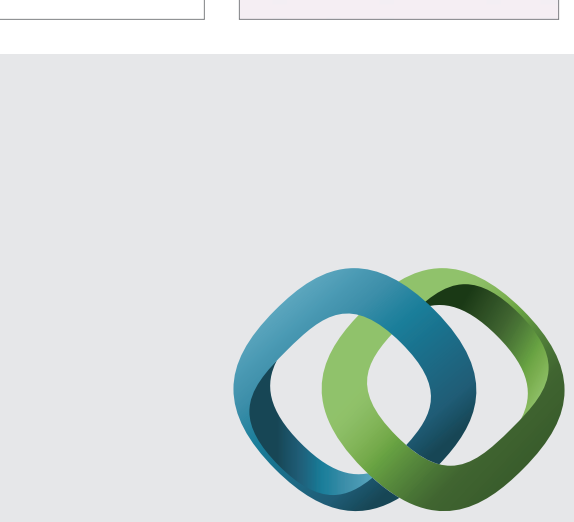

\section{Hindawi}

Submit your manuscripts at

http://www.hindawi.com
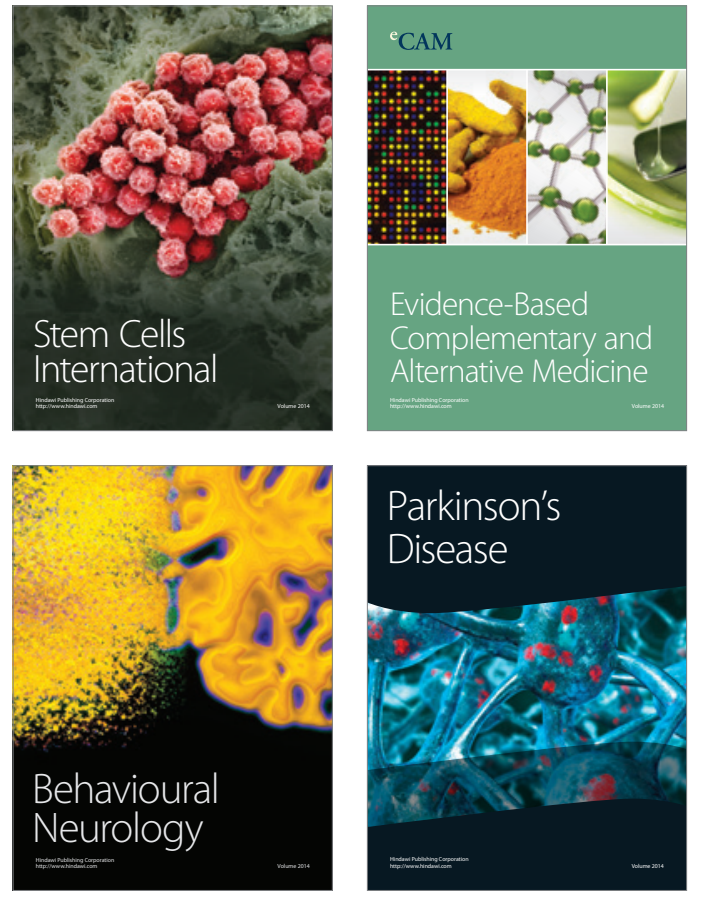
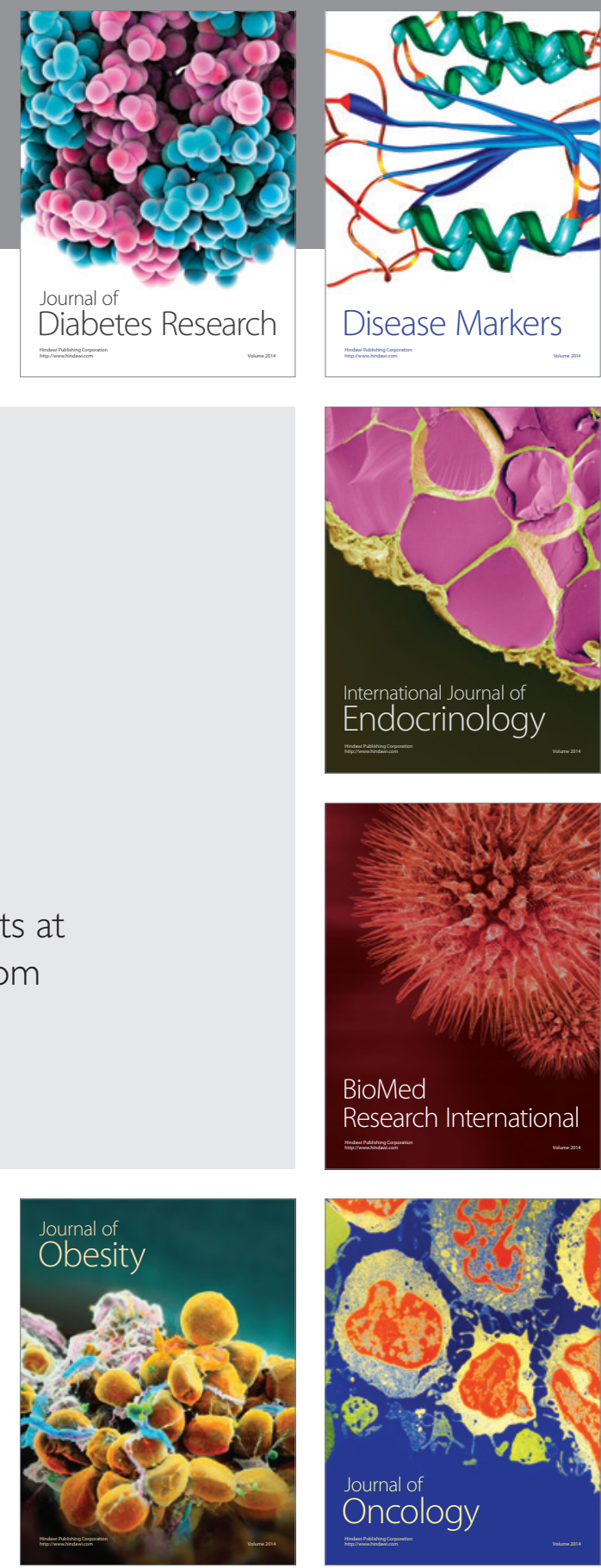

Disease Markers
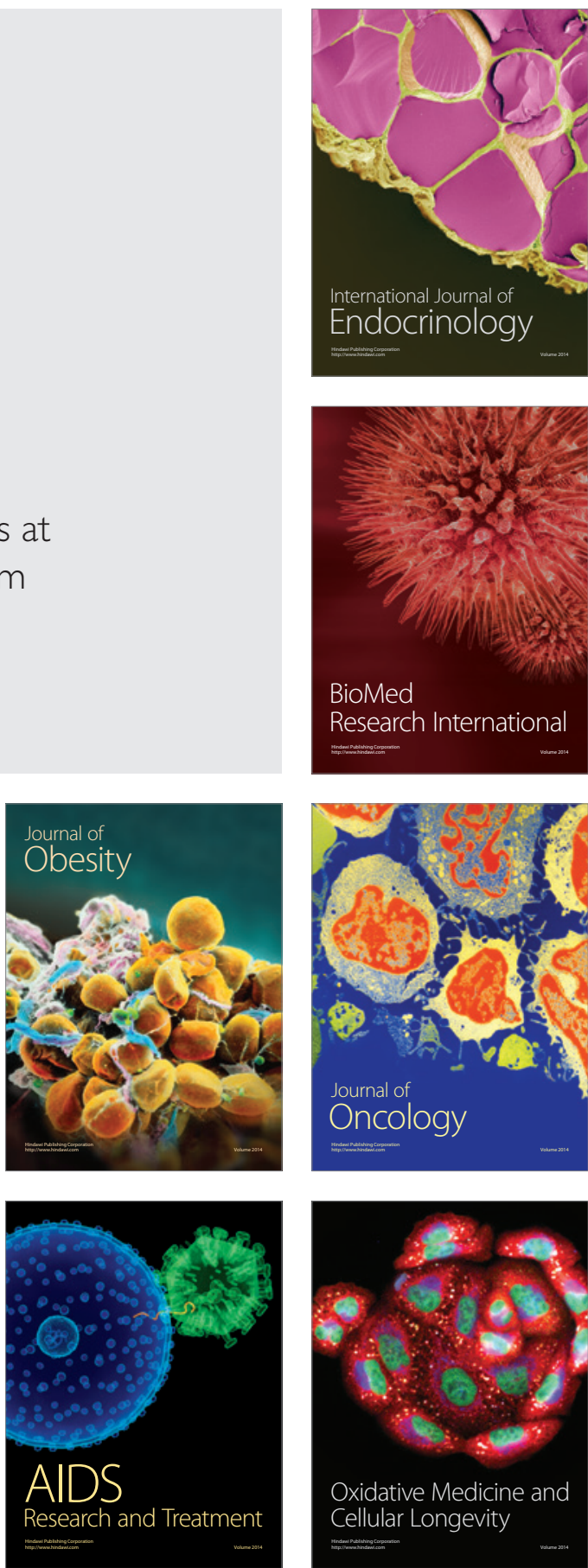